Article

\title{
On Chirality of the Vorticity of the Universe
}

\section{Davor Palle}

Division of Theoretical Physics, Rugjer Bošković Institute, Bijenička cesta 54, P.O.B. 180, HR-10002

Zagreb, Croatia; E-Mail: palle@irb.hr; Tel.: +385-1-4561-030; Fax: +385-1-4680-223

Received: 16 April 2012 / Accepted: 11 May 2012 / Published: 16 May 2012

\begin{abstract}
The presence of dark energy in the Universe challenges the Einstein's theory of gravity at cosmic scales. It motivates the inclusion of rotational degrees of freedom in the Einstein-Cartan gravity, representing the minimal and the most natural extension of the General Relativity. One can, consequently, expect the violation of the cosmic isotropy by the rotating Universe. We study chirality of the vorticity of the Universe within the Einstein-Cartan cosmology. The role of the spin of fermion species during the evolution of the Universe is studied by averaged spin densities and Einstein-Cartan equations. It is shown that spin density of the light Majorana neutrinos acts as a seed for vorticity at early stages of the evolution of the Universe. Its chirality can be evaluated in the vicinity of the spacelike infinity. It turns out that vorticity of the Universe has right-handed chirality.
\end{abstract}

Keywords: Einstein-Cartan cosmology; vorticity; Majorana neutrinos

\section{Introduction}

The two major problems in cosmology and particle physics, namely, dark energy and dark matter, force us to modify substantially our theories of basic interactions. In this paper, we show how our BY theory of [1] is compatible and closely related to the Einstein-Cartan cosmology.

To comprehend all the phenomenology in particle physics and cosmology one has to construct mathematically consistent and complete theories based on few basic physical principles. The theory presented in [1] is an attempt of solving two main obstacles in the Standard Model (SM) and relativistic quantum field theory: zero-distance singularity and causality-violating $S U(2)$ global anomaly.

The resulting theory (called BY in [1]) is UV finite (not only renormalizable) with heavy and light Majorana neutrinos as cold and hot dark matter [2,3]. There is a perfect balance between bosonic (electroweak gauge bosons) and fermionic (leptons and quarks) particles, owing to exact cancellation 
of anomalous effective actions and the constraint relation between boson and fermion mixing angles $\theta_{W}=2\left(\theta_{12}+\theta_{23}+\theta_{31}\right)$. The left-handed chirally-asymmetric weak interactions appear as an inevitable consequence of the assumed dimensionality and noncontractiblity of the physical spacetime.

The absence of Higgs particles is crucial for the cosmological stability of heavy Majorana neutrinos $\tau_{N_{i}} \gg \tau_{U}$ [2]. The lepton-number violation, the conservation of $B-L$, as well as lepton and baryon CP violation, lead to leptogenesis and baryogenesis.

To summarize, the BY gauge theory is not only mathematically superior to the SM, but also phenomenologically: (1) solar, atmospheric, neutrino and long-baseline neutrino experiments favor massive light neutrinos with mixed flavors; (2) contrary to the SM, the BY theory has heavy Majorana neutrinos as cold dark matter candidates; (3) the SM cannot generate lepto- and baryogenesis while the lepton-number violation in the BY theory together with $\mathrm{CP}$ violating phases in the quark and lepton mixing matrices allow cosmological lepto- and baryogenesis; (4) quantum-loop corrections in the electroweak and strong interactions in the SM show some deviations for the forward-backward and left-right asymmetry form factors measured by LEP2 and SLC and a difference from the QCD amplitudes at the largest momenta measured by Tevatron. The BY theory can account for these differences [4,5], however only new LHC data will select the proper symmetry-breaking mechanism. The most recent predictions of the BY theory for strong [6] (t-quark charge asymmetry) and electroweak interactions (CP violation and rare decays) $[7,8]$ are testable in the near future at hadron colliders.

Formulating the theory of the local structure of spacetime as local $S U(3) \times S U(2) \times U(1)$ gauge theories, we choose the Einstein-Cartan theory as formulated by Sciama and Kibble to be the theory of the global structure of spacetime. Trautman was the first who realized the possibility of the nonsingular Einstein-Cartan (EC) cosmology [9]. In addition, there is more freedom to avoid noncausal Goedel cosmological solutions [10,11]. The Einstein-Cartan gravity is also a quantum theory of gravity but not in a sense of introducing the spin 2 local quantum field with the corresponding Heisenberg commutation rules. The quantum principle figures only through quantum mechanical spin densities at the first quantized level.

In this paper we attempt to make a connection between the chirally asymmetric weak interactions and possibly anisotropic Universe described by the Einstein-Cartan cosmology.

\section{Theoretical Scenario}

The EC gravity relates rotational degrees of freedom of matter and spacetime, i.e., total angular momentum as a conserved quantity in the Special Theory of Relativity consisting of the orbital angular momentum and spin $[12,13]$ of matter $v$ s. torsion of spacetime. Spin, as an internal angular momentum of particles, is a quantum mechanical quantity, so it vanishes in the classical limit of the vanishing Planck constant.

One can introduce the angular momentum in General Relativity only as a nonconserved quantity and it does not obey tensorial transformations [14]. On the contrary, the EC theory of gravity incorporates spin and angular momenta of matter and torsion of spacetime invariantly with respect to the general coordinate transformations of the enlarged general theory of relativity [15-17]. Owing to the algebraic relation between spin and angular momentum vs. torsion, one can incorporate spin and angular momentum into the effective energy-momentum tensor [10,11,15-17]: 


$$
\begin{gathered}
R_{\mu \nu}-\frac{1}{2} g_{\mu \nu} R=\kappa T_{\mu \nu}^{e f f} \\
T_{\mu \nu}^{e f f}=-p_{e f f} g_{\mu \nu}+u_{\mu} u_{\nu}\left(p_{e f f}+\rho_{e f f}\right)-2\left(g^{\alpha \beta}+u^{\alpha} u^{\beta}\right) \nabla_{\alpha}\left[u_{(\mu} S_{\nu) \beta}\right], \\
\kappa=8 \pi G_{N} c^{-4}, \rho_{e f f}=\rho-\kappa S^{2}+\Lambda, p_{\text {eff }}=p-\kappa S^{2}-\Lambda, \\
S^{2}=\frac{1}{2} S_{\alpha \beta} S^{\alpha \beta}, S_{\cdot \alpha \beta}^{\mu}=u^{\mu} S_{\alpha \beta},(\alpha \beta)=\frac{1}{2}(\alpha \beta+\beta \alpha), \\
\text { torsion }=Q_{. \alpha \beta}^{\mu}, Q_{. a b}^{\mu}+2 h_{[a}^{\mu} Q_{b]}=\kappa S_{\cdot a b}^{\mu}, \\
Q_{a}=h_{a}^{\mu} Q_{\mu}, Q_{\mu}=Q_{\cdot \mu \nu}^{\nu},[\mu \nu]=\frac{1}{2}(\mu \nu-\nu \mu), \\
a, b=\text { local Lorentzian indices, } h_{a}^{\mu}=\text { tetrad basis, } \\
\eta_{a b}=\operatorname{diag}(+1,-1,-1,-1) .
\end{gathered}
$$

We denote torsion by $Q_{. \alpha \beta}^{\mu}$ and total angular momentum by $S_{. \alpha \beta}^{\mu}$.

At the weak interaction scale $R_{\text {min }}=\mathcal{O}\left(10^{-16} \mathrm{~cm}\right)$ torsion is dominated by fermion spin densities [18], while at the largest scale $R=\infty$ torsion could be dominated only by the angular momentum of the whole Universe (galaxies, groups, clusters, ...) [10,11,18]. The contribution of the torsion at present $\left(R_{0}=\mathcal{O}\left(10^{28} \mathrm{~cm}\right)\right)$ is much smaller than the mass density if the Hubble constant is small or could be much larger if the Hubble constant is large [19]. This is a consequence of the strong constraints from the age of the Universe, inevitable negative contribution to the integrated Sachs-Wolfe effect and the observed large peculiar velocities of clusters at large scales. The second scenario with large Hubble constant and large torsion at present is more probable from the theoretical and observational points of view [19]. The contribution of the torsion terms to the effective energy-momentum tensor is always negative with respect to the mass density.

The primordial mass density contrast is evolved from quantum fluctuations of the spin to the value at the photon decoupling defined by parameters of metric beyond that of Robertson-Walker [20-22].

The question posed is whether it is possible to generate a vorticity of the Universe and to fix its chirality?

The answer is positive, provided the local and global theories of spacetime are BY and EC theories.

Within this framework the evolution scenario is the following:

(1) Assuming CP violation in lepton sector, a dynamics of heavy Majorana neutrinos produces imbalance between leptons and antileptons [23] before their decoupling from primordial plasma. An example of leptogenesis generated with a Higgs mechanism and with heavy leptons can be find in the literature [24], but heavy leptons are then cosmologically unstable. The masses of heavy Majorana neutrinos in the $\mathrm{BY}$ theory are in the range from $\mathcal{O}(\mathrm{TeV})$ to $\mathcal{O}\left(10^{3} \mathrm{TeV}\right)$, thus leptogenesis happens around $T>\mathcal{O}\left(10^{3} \mathrm{TeV}\right)$.

(2) Before light Majorana neutrino decoupling $\left(T_{d e c}=f e w M e V\right)$ the imbalance in the number of baryons and antibaryons appears as a consequence of the surplus of leptons against antileptons and conserved B-L [25-27].

(3) Now follows the crucial observation: Part of the survived leptons, like electron, are produced together with neutrinos through charged current $W^{-} \rightarrow e^{-} \nu_{M}$ and the helicity $[12,13]$ of the Majorana 
neutrino $\lambda\left(\nu_{M}\right)$ is predominantly positive $\left(\lambda\left(\nu_{M}\right)=+1\right)$. This is the consequence of the two facts: (a) helicity of Dirac antineutrinos (=helicity of Majorana neutrinos) produced in $W^{-} \rightarrow e^{-} \overline{\nu_{D}}$ is positive for left-handed weak interactions and (b) production of the negative helicity relativistic Majorana neutrinos is suppressed in the same process with weak charged currents by the kinematical factor $\frac{m_{\nu}}{E} \ll 1[1,28]$. Note that the ratio of the partial decay widths of weak bosons is $\Gamma\left(W^{-} \rightarrow e^{-} \nu\right) / \Gamma(Z \rightarrow \nu \nu) \simeq 1.35$. Neutral currents do not generate imbalance in neutrino's helicities, because one relativistic neutrino of a produced pair has negative and the other one has positive helicity. Thus, irrespective of the details of the physical processes during leptogenesis, produced number $n_{+}$of neutrinos with positive helicity $\lambda\left(\nu_{M}\right)=+1 v s$. produced number $n_{-}$of neutrinos with negative helicity $\lambda\left(\nu_{M}\right)=-1$ is roughly $n_{+} / n_{-}=\mathcal{O}(10)$.

(4) It is easy to estimate number of neutrinos and other particles at the epoch of neutrino decoupling $[1,27]$ :

$$
\begin{gathered}
n_{\nu}\left(T_{0}=2.73 \mathrm{~K}\right)=\mathcal{O}\left(10^{2} \mathrm{~cm}^{-3}\right), T_{\text {dec }}(\nu)=\text { few MeV }, \\
n_{e^{-}}\left(T_{0}\right)=n_{p}\left(T_{0}\right) \simeq n_{B}\left(T_{0}\right)=\mathcal{O}\left(10^{-7} \mathrm{~cm}^{-3}\right), \\
m_{N_{i}}=\mathcal{O}(10 T e V)-\mathcal{O}(100 T e V), m_{\nu_{i}}=\mathcal{O}\left(10^{-3} \mathrm{eV}\right)-\mathcal{O}(1 \mathrm{eV}) \\
\Rightarrow \frac{n_{\nu}}{n_{e^{-}}}, \frac{n_{\nu}}{n_{N}}, \frac{n_{\nu}}{n_{B}} \gg 1, \text { at } T=\text { few } M e V
\end{gathered}
$$

We conclude that the spin density of the matter is dominated by the spin of the light neutrinos with an excess of the positive helicity states. In addition, the sum of the orbital angular momenta of particles vanishes because of the isotropy of the Universe at that moment of the evolution.

The averaging procedure for spin of cosmic fluids [29], lepton and CP violations, together with the positive helicity abundance, guarantee the nonvanishing spin, as well as nonvanishing torsion because of their algebraic relations within Einstein-Cartan equations. This asymmetry is a seed for the small vorticity with the well-defined chirality that we define later. It is clear that any cosmic observer measures different axis of vorticity, but a chirality of the vorticity is well-defined invariant quantity for any observer.

(5) The particles that can compete with neutrinos in abundances are the background photons. However, photons, as a massless gauge boson particles, do not generate the torsion in a gauge invariant way [30].

(6) Electromagnetic and strong forces, as well as the Riemann curvature of spacetime induced by the metric alone, are chirally-symmetric interactions and cannot alter the chirality of vorticity in later stages of evolution after neutrino decoupling.

Vorticity induced by spin density within EC cosmology (see equations below) acts as a seed for vortical motions of cosmic particles and as a seed for the angular momenta of galaxies, clusters,..., Universe [27,31,32]. Torsion grows together with a grow of large scale structures at late stages of the structure formation because the torsion is then dominated by the orbital angular momenta of large scale structures.

(7) A term of EC equations linear in torsion (angular momentum) allows us to uniquely determine chirality of the vorticity. Namely, this is possible to achieve because of the relation derived at 
spacelike infinity and its vicinity [18,33]. The present Universe is in the matter dominated epoch with $T_{\gamma, 0}=2.73 \mathrm{~K}, \frac{\rho_{\gamma, 0}}{\rho_{m, 0}}=\mathcal{O}\left(10^{-4}\right)$, while the spacelike infinity is at $T_{\gamma}(R=\infty)=0 \mathrm{~K}$. Evidently, the present state of the Universe is in the vicinity of the spacelike infinity.

We define the metric with vorticity with two real parameters $m, \Sigma[11,18]$, spin (ang. momentum) and torsion of the fluid [11], as well as the standard definition of vorticity [18]. The crucial relations (Equations 3) at spacelike infinity allows to fix the chirality of the vorticity from a chirality of the angular momentum:

$$
\begin{aligned}
& \text { by definition : } S^{\alpha \beta}=-\frac{1}{2} n h_{i}^{\alpha} h_{j}^{\beta} \mu^{i j} \text {, } \\
& \text { metric : } d s^{2}=d t^{2}-d x^{2}-(1-\Sigma) e^{2 m x} d y^{2}-d z^{2}-2 \sqrt{\Sigma} e^{m x} d y d t \text {, } \\
& \Sigma, m=\text { constant parameters, } \\
& R=\infty: Q=Q_{. \hat{1} \hat{2}}^{\hat{0}}=-\frac{m(2-\Sigma)}{2 \sqrt{\Sigma}}, \Sigma=\mathcal{O}\left(10^{-3}\right) \ll 1 \\
& G_{N} \rho_{\infty} H_{\infty}^{-2}=\frac{3}{4 \pi}, H_{\infty}=\text { Hubble constant at } \infty, \\
& \text { by definition : } Q=\frac{1}{2} \kappa n \mu^{\hat{2} \hat{1}} B_{\infty}, \mu^{\hat{1} \hat{2}}=-\mu^{\hat{2} \hat{1}}=+\frac{1}{2} \hbar \text {, } \\
& n=n_{\nu}(\lambda=+1)-n_{\nu}(\lambda=-1)>0 .
\end{aligned}
$$

The asymmetry in the lepton or baryon numbers is large from the beginning of the activation of $\mathrm{CP}$ violating processes by weak interactions. $B_{\infty}$ is some positive amplification factor as a result of the evolution. Its magnitude can be estimated $B_{\infty} \simeq Q(\text { Universe })_{0} / \kappa \operatorname{Spin}(\text { neutrinos })_{0}=\mathcal{O}\left(10^{34}\right)$. This is a consequence of the fact that the torsion of the Universe $Q$ (Universe $)_{0}$ at present time is predominantly defined by its macroscopical orbital angular momentum and it cannot vanish because of the nonvanishing primordial vorticity. From preceding equations and [18], it follows $\left|Q_{0}\right| \simeq \sqrt{3} H_{0}$, therefore the orbital angular momentum of the Universe can be estimated to be $L_{U} \simeq \frac{1}{\sqrt{3}} G_{N}^{-1} H_{0}^{-2}=$ $2 h^{-2} \times 10^{94} \mathrm{gcm}^{2} \mathrm{~s}^{-1}$.

It is advantageous to check the estimate of the angular momentum of the Universe by the relation of the rotational support [34] (rotation against gravity) $L=\sigma \frac{G_{N} M^{5 / 2}}{|E|^{1 / 2}}$, where $\sigma$ is a dimensionless spin parameter, $\mathrm{M}$ the mass and $\mathrm{E}$ the energy of the physical system $|E| \simeq \frac{G_{N} M^{2}}{R}$. Acknowledging that $R_{U}=H_{0}^{-1}, M_{U}=\Omega_{m} \rho_{c} \frac{4 \pi}{3} R_{U}^{3}=\frac{\Omega_{m}}{2} G_{N}^{-1} H_{0}^{-1}$, one easily gets $L_{U}=\sigma\left(\frac{\Omega_{m}}{2}\right)^{3 / 2} G_{N}^{-1} H_{0}^{-2}$. Rotational support requires that $\sigma=\mathcal{O}(1)$, while in the EC cosmology $\Omega_{m}=2$ [18], thus the estimate for $L_{U}$ agrees with a previous one derived in the EC cosmology.

Now, we acknowledge the fact of the abundant positive-helicity states of neutrinos contributing to the spin density and leading to the positive chirality angular momentum of the Universe. It follows then from Equation (3)

$$
Q<0 \Rightarrow m>0 \text {. }
$$


Definition and chirality of vorticity are naturally defined as [35-37] (standard right-handed xyz frame is assumed):

$$
\begin{gathered}
\omega_{\nu \mu}=\frac{1}{2}\left(\nabla_{\alpha} u_{\beta}-\nabla_{\beta} u_{\alpha}\right) P_{\mu}^{\alpha} P_{\nu}^{\beta}, \\
P_{\alpha \beta}=g_{\alpha \beta}-u_{\alpha} u_{\beta}, \omega_{i j}=h_{i}^{\mu} h_{j}^{\nu} \omega_{\mu \nu}, \\
\omega^{\nu \mu}=h_{i}^{\nu} h_{j}^{\mu} \omega^{i j} \\
\Sigma H_{\infty} \simeq \frac{2}{\sqrt{3}} \omega_{\infty}, \omega_{\infty}^{2}=\frac{1}{2} \omega_{\mu \nu} \omega^{\mu \nu}, \\
\omega_{\mu \nu}(m=+|m|, x y z)=-\omega_{\mu \nu}(m=-|m|, x y z)=-\omega_{\mu \nu}(m=+|m|, \text { yxz }), \\
m>0 \text { and } \omega_{\hat{1} \hat{2}}=\omega^{\hat{1} \hat{2}}=+m \frac{\sqrt{\Sigma}}{2}>0 \Rightarrow \text { right }- \text { handed vorticity }
\end{gathered}
$$

Hence, if the abundant positive-definite helicity light neutrinos define the torsion's chirality, then the chirality of the vorticity of the Universe is right-handed, i.e., it is positive in the standard right-handed frame.

\section{Conclusions and Remarks}

The magnitude and chirality of the vorticity and angular momentum of the Universe, as well as the relic-neutrino helicities, are well-defined observables. Numerous statistical studies of the WMAP data reported a violation of the isotropy of the Universe, while analyses of clusters found anisotropic large scale flows (see references in [19]).

Dark energy is, in our scenario, the angular momentum of the Universe, giving the negative contribution to the effective mass density of the Universe (from Equation 3: $\Omega_{m}=2, \Omega_{Q}=-1$ and $\Omega_{\Lambda}=0$, because $\lim _{R \rightarrow \infty} \rho_{\Lambda}=-\frac{1}{2} \lim _{R \rightarrow \infty} \rho_{m}=0$ ). Note that in the quasilinear regime, at the galaxy forming epoch, $\rho_{Q} \propto(1+z)^{3}$ [19], while in the nonlinear galaxy-cluster forming epoch (at very low redshifts), one expects $\rho_{Q} \propto$ const [34].

The existence of the lepton CP violation is indispensable [23] in our cosmological scenario with BY and EC theories. Recent results of MiniBooNE and MINOS experiments with different oscillation results for neutrinos and antineutrinos strongly suggest possible lepton CP violation if the data are fitted with three neutrino flavors. Note that the $\mathrm{CP}$ violating phase need not to be accompanied with $\theta_{31}$ mixing angle, but can be attached to $\theta_{12}$ mixing angle. The inclusion of large $\mathrm{CP}$ violating phase can substantially change the present estimate of the mixing angles.

We see that the chirality of the asymmetry in particle physics is left-handed (weak interactions), thus opposite to the positive chirality of the vorticity of the Universe, all measured in the right-handed coordinate reference frames. If we change a reference frame to be left-handed, the chiralities change signs. The sum of chiralities of the microstructure and the macrostructure of the physical world remains zero irrespective of our choice of the reference frame. Parity violation in particle physics and cosmology is mandatory from both physical and mathematical points of view. The PLANCK mission can give us a definitive answer on the chirality of the vorticity of the Universe. 


\section{References}

1. Palle, D. On the broken gauge, conformal and discrete symmetries in particle physics. Nuovo Cimento A 1996, 109, 1535-1554.

2. Palle, D. On the lifetime of a cold dark matter particle and the cosmological diffuse photon background. Nuovo Cimento B 2000, 115, 445-458.

3. Palle, D. On heavy Majorana neutrinos as a source of the highest energy cosmic rays. Nuovo Cimento B 2003, 118, 747-752.

4. Palle, D. On the enhancement of the QCD running coupling in the noncontractible space and anomalous Tevatron and HERA data. Hadronic J. 2001, 24, 87-102.

5. Palle, D. On the quantum loop weak interaction corrections at high energies. Hadronic J. 2001, 24, 469-484.

6. Palle D. On the anomalous t-quark charge asymmetry and noncontractibility of the physical space, preprint. arXiv 2012, arXiv:1204.1171.

7. Palle, D. On the anomalous CP violation and noncontractibility of the physical space, preprint. arXiv 2011, arXiv:1111.1638.

8. Palle, D. On the rare $B_{s} \rightarrow \mu \mu$ decay and noncontractibility of the physical space, preprint. arXiv 2011, arXiv:1111.1639.

9. Trautman, A. Spin and torsion may avert gravitational singularities. Nature 1973, 242, 7-8.

10. Ray, J.R.; Smalley, L.L. Improved perfect-fluid energy-momentum tensor with spin in Einstein-Cartan space-time. Phys. Rev. Lett. 1982, 49, 1059-1061.

11. Obukhov, Yu.N.; Korotky, V.A. The Weyssenhoff fluid in Einstein-Cartan theory. Classical Quant. Grav. 1987, 4, 1633-1657.

12. Bjorken J.D.; Drell S.D. Relativistic Quantum Fields; McGraw-Hill Book Company: New York, NY, USA, 1965.

13. Bogoliubov, N.N.; Shirkov, D.V. Vvedenie v teoriju kvantovannih poljei (in Russian); Izdateljstvo Nauka: Moscow, Russia, 1973.

14. Weinberg, S. Gravitation and Cosmology; J. Wiley and Sons: New York, NY, USA, 1972.

15. Trautman, A. On the structure of the Einstein-Cartan equations. Sym. Math. 1973, 12, 139-160.

16. Hehl, F.W. Spin and torsion in general relativity: I. Foundations. Gen. Relat. Gravit. 1973, 4, 333-349.

17. Hehl, F.W. Spin and torsion in general relativity: II. Geometry and Field Equations. Gen. Relat. Gravit. 1974, 5, 491-516.

18. Palle, D. On certain relationships between cosmological observables in the Einstein-Cartan gravity, Nuovo Cimento B 1996, 111, 671-675.

19. Palle, D. On the anomalous large scale flows in the Universe. EPJ C 2010, 69, 581-589.

20. Palle, D. On primordial cosmological density fluctuations in the Einstein-Cartan gravity and COBE data. Nuovo Cimento B 1999, 114, 853-860.

21. Palle, D. On the evolution of the cosmic-mass-density contrast and the cosmological constant. Nuovo Cimento B 2006, 121, 129-136. 
22. Palle, D. Breaking the scale invariance of the primordial spectrum or not: The new WMAP data. Nuovo Cimento B 2007, 122, 67-73.

23. Sakharov, A.D. Violation of CP invariance, C asymmetry, and baryon asymmetry of the universe. JETP Lett. 1967, 5, 24-27.

24. Luty, M. Baryogenesis via Leptogenesis. Phys. Rev. D 1992, 45, 455-465.

25. 't Hooft, G. Symmetry breaking through Bell-Jackiw anomalies. Phys. Rev. Lett. 1976, 37, 8-11.

26. Kuzmin, V.A.; Rubakov, V.A.; Shaposhnikov, M.E. On anomalous electroweak baryon-number non-conservation in the early Universe. Phys. Lett. B 1985, 155, 36-42.

27. Kolb, E.W.; Turner, M.S. The Early Universe; Addison-Wesley: Redwood City, CA, USA, 1990.

28. Kayser, B.; Gibrat-Debu, F.; Perrier, F. The Physics of Massive Neutrinos; World Scientific: Singapore, 1989.

29. Hehl, F.W.; von der Heyde, P.; Kerlick, G.D. General relativity with spin and torsion and its deviations from Einstein's theory. Phys. Rev. D 1974, 10, 1066-1069.

30. Hayashi, K.; Bregman, A. Poincaré Gauge invariance and the dynamical role of spin in gravitational theory. Ann. Phys. (N.Y.) 1973, 75, 562-600.

31. Peebles, P.J.E. The Large-Scale Structure of the Universe; Princeton University Press: Princeton, NJ, USA, 1980.

32. Binney, J.; Tremaine, S. Galactic Dynamics; Princeton University Press: Princeton, NJ, USA, 1994.

33. Penrose, R. Zero rest-mass fields including gravitation: Asymptotic behavior. Proc. Roy. Soc. Lond. Math. Phys. Sci. 1965, 284, 159-203.

34. Padmanabhan, T. Structure Formation in the Universe; Cambridge University Press: Cambridge, UK, 1995.

35. Bedran, M.L.; Vasconcellos-Vaidya, E.P.; Som, M.M. Stationary cosmological solution with torsion. Nuovo Cimento B 1985, 87, 101-108.

36. Ehlers, J. Contributions to the relativistic mechanics of continuous media. Gen. Relat. Gravit. 1993, 25, 1225-1266.

37. Kreyszig, E. Differentialgeometrie; Akademische Verlagsgesellschaft Geest und Portig: Leipzig, Germany, 1957.

(c) 2012 by the author; licensee MDPI, Basel, Switzerland. This article is an open access article distributed under the terms and conditions of the Creative Commons Attribution license (http://creativecommons.org/licenses/by/3.0/.) 